\title{
Riemann-Liouville Fractional Operators of Bicomplex Order and Its Properties.
}

\author{
Mahesh Goswami ${ }^{1}$ and Raj Kumar ${ }^{1}$ \\ ${ }^{1}$ Mohanlal Sukhadia University Department of Mathematics
}

May 22, 2021

\begin{abstract}
In this paper, we construct the Riemann-Liouville fractional integral and differential operator of bicomplex order and illustrate some examples to calculate the fractional integration and differentiation of bicomplex order of some elementary functions. Also, we discuss some properties of these operators by proving analogues of the Leibniz and chain rules for these operators.
\end{abstract}

\section{Hosted file}

Riemann-Liouville Frcational Operators of Bicomplex order and Its Proerties..pdf available at https://authorea.com/users/415124/articles/523037-riemann-liouville-fractionaloperators-of-bicomplex-order-and-its-properties

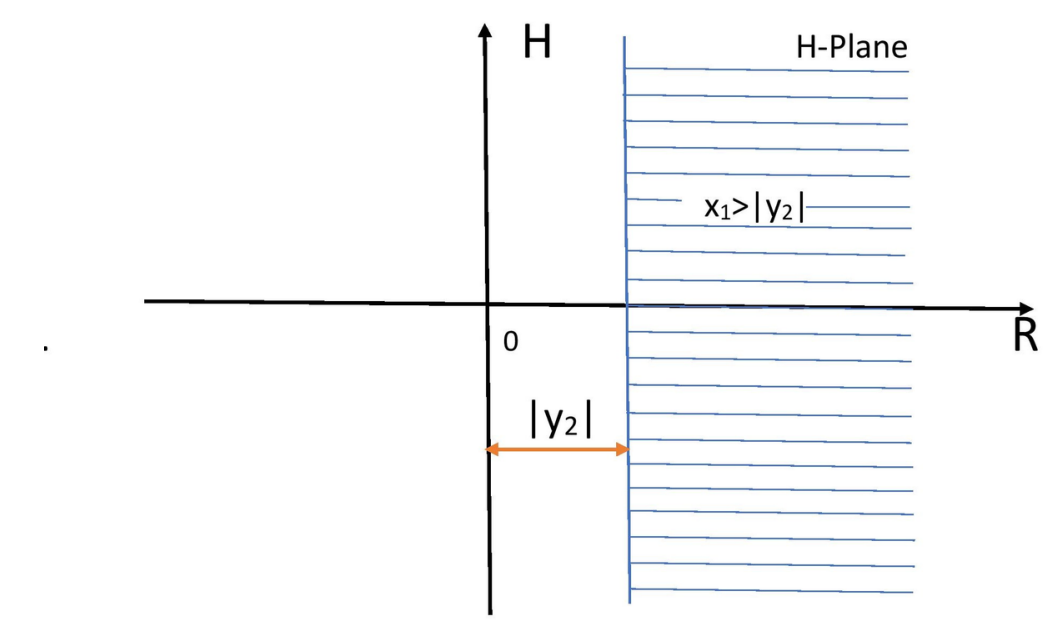

Figure : 1 


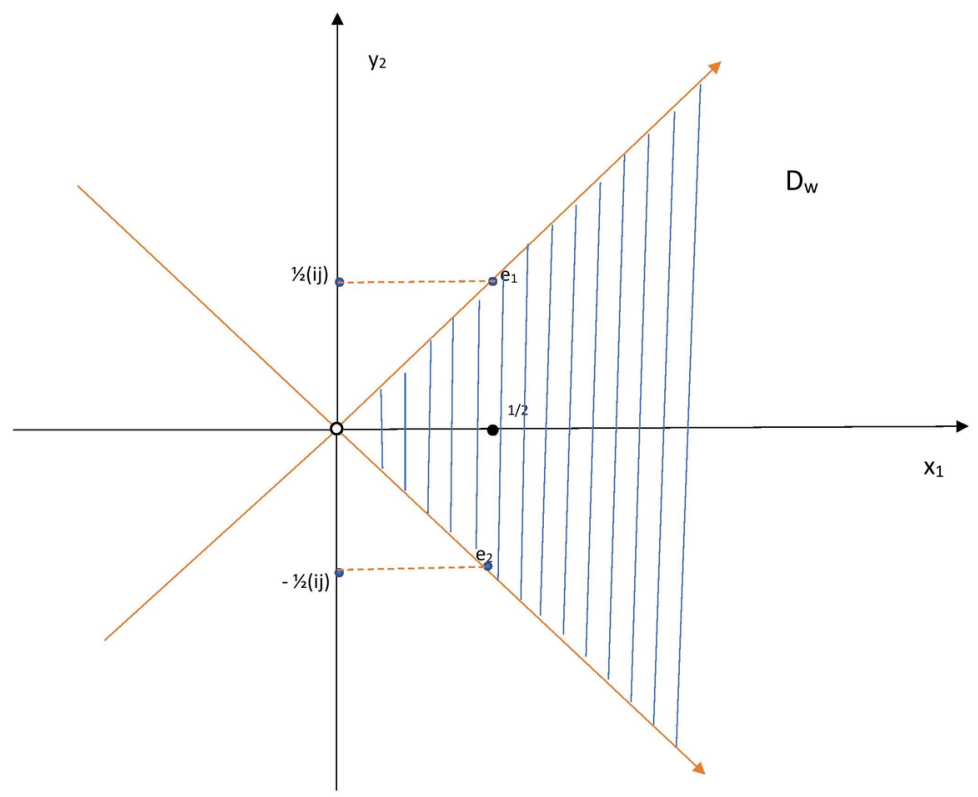

Figure: 2 